\title{
Ultrasound Imaging Of The Knee Showing A Fortuitous Calcification In The Lateral Collateral Ligament
}

\author{
Levent Özçakar' ${ }^{\text {, Naser B. Albarazi², Ahmad J. Abdulsalam² }}{ }^{2}$
}

${ }^{1}$ Department of Physical and Rehabilitation Medicine, Hacettepe University Medical School, Ankara, Turkey, ${ }^{2}$ Department of Physical Medicine and Rehabilitation Physical Medicine and Rehabilitation Hospital, Andalous, Kuwait

\section{To the Editor,}

A 47-year-old man had been seen for bilateral knee pain for the last 3 months. His medical history revealed multiple sclerosis for 15 years and an arthroscopic menisectomy for the right knee 12 years before presentation. Physical examination was unremarkable. On ultrasound (US) imaging, the right medial meniscus was observed to be absent and there was a small calcification in the proximal attachment site of the left lateral collateral ligament (LCL) (fig 1). Sonopalpation did not yield any pain. Accordingly, in addition to 'sonographic reassurance' with regard to his knee findings and suggestions to lose weight, the patient was prescribed a home-based exercise regimen for strengthening the knee extensors.

Due to its anatomical alignment (with steep angulation), lateral collateral ligament of the knee is not commonly scanned in each/every knee US protocol [1]. This is also true for menisci and cruciate ligaments. However, scanning these structures with US is very convenient and can be conclusive in particular cases. Likewise, in terms of calcification, it can be also be the first imaging tool which is able detect the ectopic calcification even when radiography and/or computed tomography are negative [2]. To this end, especially in a case of painless LCL calcification - which is quite uncommon in the literature [3] - this physician and patient friendly technique can be readily used (as the extension of a physical examination) for 'sonographic' reassurance. However, the eventual medical

Received 18.03.2019 Accepted 02.04.2019

Med Ultrason

2019, Vol. 21, No 2, 204, DOI: 10.11152/mu-1954

Corresponding author: Ahmad J. Abdulsalam

Department of Physical Medicine and Rehabilitation, Physical Medicine and Rehabilitation Hospital, Andalous, Kuwait E-mail: Dr.ahmad.j.abdulsalam@gmail.com

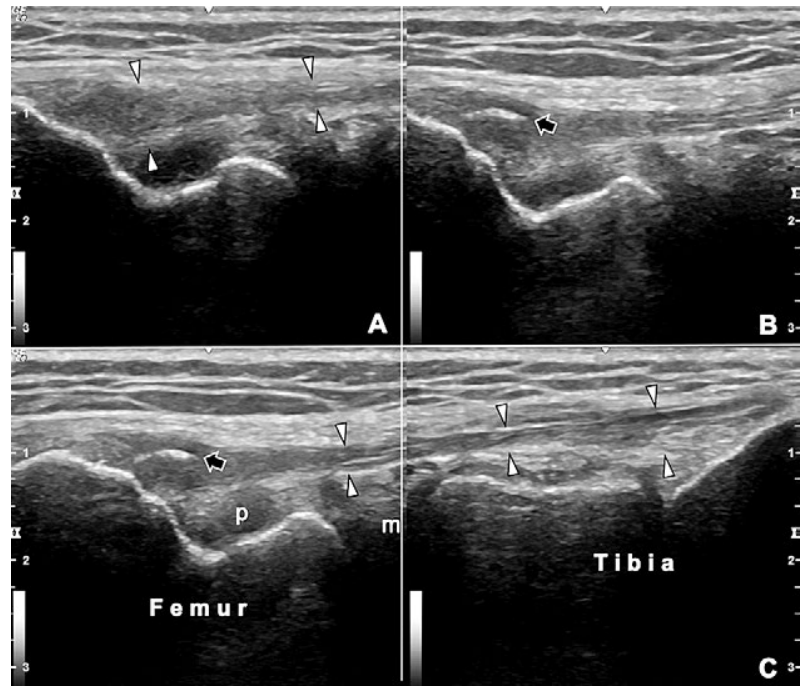

Fig 1. Comparative knee US imaging (lateral longitudinal view) shows the calcification in the proximal attachment site of the LCL overlying the notch where the popliteus tendon takes off. Right knee (A), left knee (B) and split screen view of the left knee (C). m: lateral meniscus, p: popliteus tendon (anisotropy)

decision (how to treat the patient) should be based on the symptomatology, clinical and imaging findings.

\section{References}

1. Özçakar L, Kara M, Chang KV, et al. EURO-MUSCULUS/ USPRM. Basic scanning protocols for knee. Eur J Phys Rehabil Med 2015;51:641-646.

2. Özçakar L, Carli AB, Tok F, Tekin L, Akkaya N, Kara M. The utility of musculoskeletal ultrasound in rehabilitation settings. Am J Phys Med Rehabil 2013;92:805-817.

3. Anderson SE, Bosshard C, Steinbach LS, Ballmer FT. MR imaging of calcification of the lateral collateral ligament of the knee: a rare abnormality and a cause of lateral knee pain. AJR Am J Roentgenol 2003;181:199-202. 


\title{
Comment on "Visual versus automatic ultrasound scoring of lung B-lines: reliability and consistency between systems"
}

\section{Count of B-lines: a reappraisal}

\author{
Carla Maria Irene Quarato¹, Donato Lacedonia1, Anna Del Colle, Giulia Gaudiuso², \\ Elisabettamaria Frongillo ${ }^{3}$, Cristiana Cipriani ${ }^{4}$, Maria Giulia Tinti ${ }^{5}$, Marco Sperandeo ${ }^{6}$
}

\begin{abstract}
${ }^{1}$ Department of Respiratory Disease, University of Foggia, ${ }^{2}$ Department of Respiratory Disease, University of Bari, ${ }^{3}$ Unit of Thoracic Surgery IRCCS Fondazione Casa Sollievo della Sofferenza, San Giovanni Rotondo, ${ }^{4}$ Department of Internal Medicine and Medical Disciplines, Sapienza University of Rome, ${ }^{5}$ Unit of Geriatric IRCCS Fondazione Casa Sollievo della Sofferenza, San Giovanni Rotondo, ${ }^{6}$ Unit of Interventional and Diagnostic Ultrasound of Internal Medicine IRCCS Fondazione Casa Sollievo della Sofferenza, San Giovanni Rotondo, Italy
\end{abstract}

\section{To the Editor,}

We read with great interest the article by Short et al on visual versus automatic ultrasound scoring of lung Blines [1] and we feel we must take issue with some of the comments published. B-lines are considered by the authors as "US signs of thickening of interlobular septa due to the presence of increased fluid or collagen tissue alteration". Actually, the generation of US artifacts mainly depends on the high difference in acoustic impedance that the ultrasounds encounter when they cross surfaces with a different density (i.e. chest wall/aerated lung and fluid film), resulting in a reflection of more than $96 \%$ of the ultrasound beam at the tissue/air interface and in the generation of a hyperechoic pleural line without a real anatomic match, and also in physical artifacts of reverberation: B-lines (or ring-down) and A-lines (simple reverberations) [2]. B-lines artifacts generate when sound waves interact with gas bubbles, exciting the fluid trapped between the bubbles and causing the fluid to resonate [3]. That is why the B-lines are present also in the bowel loops [4] and in the residual cavity of the postpneumonectomy space [5], while they are absent during intraoperatory ultrasound scans, also in patients with

Received 24.02.2019 Accepted 10.03.2019

Med Ultrason

2019, Vol. 21, No 2, 205-206, DOI: 10.11152/mu-1930

Corresponding author: Carla Maria Irene Quarato

Department of Respiratory Disease,

University of Foggia, Foggia, Italy

E-mail: c.quarato@libero.it

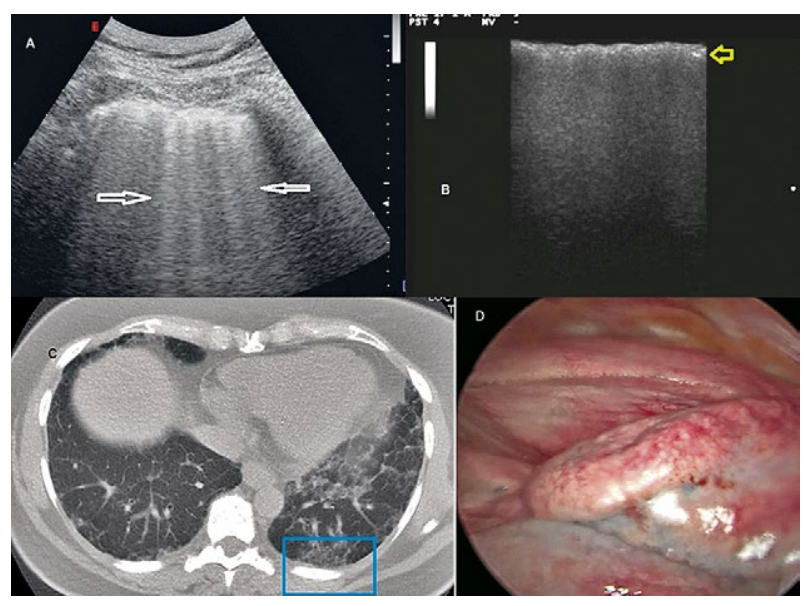

Fig 1. A: Transthoracic ultrasound image showing irregular thickening of the hyperechoic pleural line $(5 \mathrm{~mm})$ and $\mathrm{B}$ line below (white arrows). B: Video-assisted thoracic surgery ultrasound (VATS-US) pattern of increased thickness of the pleural line (arrow blue) with no artifact below; C: High resolution chest tomography showing undefined lung fibrosis (corresponding Transthoracic ultrasound scan in box blue) D: VATS image of patient with Non Specific Interstitial Pneumonia (histologic diagnosis with biopsy during VATS).

pulmonary fibrosis [6] (fig 1), according to the nature of physical effects. Moreover, the number of ultrasound artifacts may vary depending on the machine set-up, the type of probe (linear, convex or sectorial) and the patient's position [2] . In addition, TUS can, at best, explore about $70 \%$ of the pleural surface [2]. Why did the authors choose not to scan the back lung? In conclusion, in our opinion, since it is not possible to define an accurate 
correlation between the number of lines B and the presence of a specific underlying pulmonary disease, there is doubt in the validity of counting the number of lines B as a sign of nonspecific pathology, regardless of whether visually or by an automatic counting of the ultrasound device.

\section{References}

1. Short J, Acebes C, Rodriguez-de-Lema G, et al. Visual versus automatic ultrasound scoring of lung B-lines: reliability and consistency between systems. Med Ultrason 2019;21:45-49.

2. Sperandeo M, Rotondo A, Guglielmi G, Catalano D, Feragalli B, Trovato GM. Transthoracic ultrasound in the as- sessment of pleural and pulmonary diseases: use and limitations. Radiol Med 2014;119:729-740.

3. Dogra V, Rubens DJ. (eds.). Ultrasound Secrets. 1st ed. Philadelphia: Hanley \& Belfus; 2004.

4. Wilson SR, Burns PN, Wilkinson LM, Simpson DH, Muradali D. Gas at Abdominal US: Appearance, Relevance, and Analysis of Artifacts. Radiology 1999;210:113123.

5. Cavaliere F, Zamparelli R, Soave MP, Gargaruti R, Scapigliati A, De Paulis S. Ultrasound artifacts mimicking pleural sliding after pneumonectomy. J Clin Anesth 2014;26:131-135.

6. Tinti MG, Frongillo E, Sperandeo M. Lung Fissures Detection With Transthoracic Ultrasound: Is It Simply a Matter of Artifacts? Chest 2018;154:453-455.

\section{Author's response}

\section{Jorge Short ${ }^{1}$, Carlos Acebes ${ }^{2}$, Guido Rodriguez-de-Lema ${ }^{1}$, Giuliana Maria Concetta La Pagliaa ${ }^{3}$, Maria Pavón ${ }^{1}$, Olga Sánchez-Pernaute ${ }^{3}$, Julio Cesar Vazquez ${ }^{1}$, Fredeswinda Romero-Bueno ${ }^{3}$, Jesús Garrido ${ }^{5}$, Esperanza Naredo ${ }^{3}$}

${ }^{1}$ Department of Emergency Medicine, Ultrasound Division. Hospital Universitario Fundación Jiménez Díaz, IIS Fundación Jiménez Díaz and Universidad Autónoma de Madrid. Madrid, Spain, ${ }^{2}$ Rheumatology Unit, Hospital General de Villalba. Madrid, Spain, ${ }^{3}$ Department of Rheumatology, Bone and Joint Research Unit. Hospital Universitario Fundación Jiménez Díaz, IIS Fundación Jiménez Díaz, and Universidad Autónoma de Madrid. Madrid, Spain, ${ }^{4}$ Division of Rheumatology and Clinical Immunology, Humanitas Clinical and Research Center, Rozzano, Italy, ${ }^{5}$ Department of Social Psychology and Methodology, Facultad de Psicología, Universidad Autónoma de Madrid, Spain

\section{Dear Editor,}

We thank Quarato et al for their interest in our study and their constructive comments. Indeed, as Quarato et al elegantly described, ultrasound (US)-detected lung B-lines are produced by a change in the physical properties of the lung $[1,2]$. These US artefacts are considered as a surrogate marker of alveolar-interstitial pathological changes in a variety of lung conditions [3-5]. We agree that there is currently no evidence that the number of lines B correlates with a specific pulmonary disease. We also agree that the detection of lung B-lines may be influenced by the US settings, scanning technique, and patient's position. Due to the latter, standardization of lung US in any medical discipline is extremely important. On the other hand, as we have acknowledged in our discussion, we chose a reduced intercostal space evalu- ation to make the exercise feasible and friendly for the patients. Last but not least, although only a few data are available yet on the responsiveness of US-detected B lines in rheumatoid arthritis and connective tissue diseases [6,7], there is evidence supporting the use of lung US for monitoring pulmonary decongestion after diuretic therapy in heart failure $[8,9]$. We therefore consider that B-line counting makes sense and has a value in clinical practice. In any case, the objective of our study was to assess the reliability and agreement between the classical visual counting and a new automatic counting system of lung B-lines.

\section{References}

1. Soldati G, Inchingolo R, Smargiassi A, et al. Ex vivo lung sonography: morphologic-ultrasound relationship. Ultrasound Med Biol 2012;38:1169-79. 
2. Soldati G, Demi M, Inchingolo R, Smargiassi A, Demi L. On the physical basis of pulmonary sonographic interstitial syndrome. J Ultrasound Med 2016;35: 2075 86.

3. Jambrik Z, Monti C, Coppola V, Mottola G, Miniati M, Picano E. Usefulness of ultrasound comets as a nonradiologic sign of extravascular lung water. Am J Cardiol 2004;93:1265-70.

4. Agricola E, Bove T, Oppizzi M, et al. "Ultrasound comettail images": a marker of pulmonary edema: a comparative study with wedge pressure and extravascular lung water. Chest 2005;127:1690-95.

5. Soldati G, Copetti R, Sher S. Sonographic interstitial syndrome: the sound of lung water. J Ultrasound Med 2009;28:163-74.
6. Laria A, Lurati A, Scarpellini M. Ultrasound in rheumatologic interstitial lung disease: a case report of nonspecific interstitial pneumonia in rheumatoid arthritis. Case Rep Rheumatol 2015;2015:107275.

7. Buda N, Kosiak W, Smolenska Z, Zdrojewski Z. Transthoracic lung ultrasound in the monitoring of interstitial lung disease: a case of scleroderma. Pol Arch Med Wewn 2013;123:721-2.

8. Gargani L. Ultrasound of the Lungs: More than a Room with a View. Heart Fail Clin 2019;15:297-303.

9. Öhman J, Harjola VP, Karjalainen P, Lassus J. Assessment of early treatment response by rapid cardiothoracic ultrasound in acute heart failure: Cardiac filling pressures, pulmonary congestion and mortality. Eur Heart J Acute Cardiovasc Care 2018;7:311-20. 